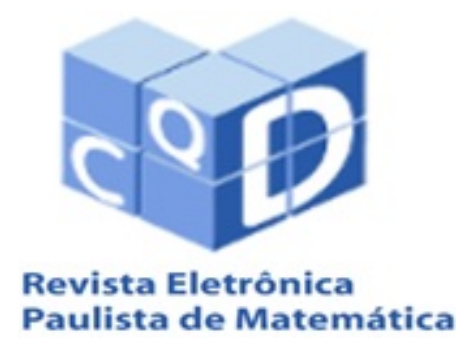

ISSN 2316-9664

Volume 10, dez. 2017

Edição Ermac

Iniciação Científica

\section{Carina Alves}

UNESP - Universidade Estadual

Paulista "Júlio de Mequita

Filho", Rio Claro

carina@rc.unesp.br

\section{Cintya Wink de Oliveira \\ Benedito}

UNESP - Universidade Estadual

Paulista "Júlio de Mequita

Filho", São João da Boa Vista

cintya.benedito@sjbv.unesp.br

\section{William Lima da Silva Pinto}

UNESP - Universidade Estadual

Paulista "Júlio de Mequita

Filho", Rio Claro

william26535@hotmail.com

\section{Reticulados via polinômios de grau 2 e 3}

Lattices via polynomials of degree 2 and 3

\section{Resumo}

A teoria de reticulados algébricos têm ganhado destaque nos últimos anos devido às aplicações na Teoria da Informação, mais especificamente, constelações de sinais tendo uma estrutura de reticulados tem sido usada como suporte para a transmissão de sinais sobre os canais gaussiano e com desvanecimento do tipo Rayleigh. O problema de encontrar boas constelações de sinais para um canal gaussiano está associado à procura por reticulados com alta densidade de empacotamento, que é a proporção do espaço $\mathbb{R}^{n}$ coberta pela união de esferas de mesmo raio de forma que a intersecção de quaisquer duas esferas tenha no máximo um ponto. Os reticulados de maior densidade de empacotamento são conhecidos apenas nas dimensões 1 a 8 e 24 . Assim, neste trabalho propomos uma construção de reticulados que tem a melhor densidade de empacotamento nas dimensões 2 e 3 . Para isso, fazemos o uso de polinômios de grau 2 e 3 sobre $\mathbb{Q}$.

Palavras-chave: Reticulados. Polinômios. Densidade de empacotamento. Norma mínima. Raízes.

\begin{abstract}
The algebraic lattice theory has won featured in recent years due to the applications in the Information Theory, more specifically, signal constellations having a lattice structure have been used as support for transmitting signals over the Gaussian and Rayleigh fading channels. The problem of finding good constellations of signals to a Gaussian channel is associated with the search for lattices with high packing density, which is the proportion of the space $\mathbb{R}^{n}$ covered by the union of spheres of the same radius so that the intersection of any two spheres has a maximum of one point. The lattices having the highest packing density are known only in the dimensions 1 to 8 and 24. Thus, in this work we propose a construction of lattices that have the best packing density in dimensions 2 and 3 . For this, we make use of polynomials of degree 2 and 3 over $\mathbb{Q}$.
\end{abstract}

Keywords: Lattices. Polynomials. Packing density. Minimum norm. Roots. 


\section{Introdução}

Um dos parâmetros para se encontrar bons códigos corretores de erros está ligado ao problema do empacotamento de esferas, que surgiu a partir do $18^{\circ}$ Problema de Hilbert, que é uma forma de dispor esferas no espaço euclidiano de modo a cobrir a maior parte do espaço. Este problema é denominado de empacotamento esférico, e quando o conjunto de centros das esferas formam um subgrupo discreto do $\mathbb{R}^{n}$, estes empacotamentos passam a se chamar empacotamentos reticulados. A partir daí, passaram a associar o estudo dos códigos aos reticulados e surgiram várias famílias de reticulados. Dentre tais famílias destaca-se a família dos reticulados que são obtidos via o homomorfismo de Minkowski. Pode-se mostrar que a imagem de tal homomorfismo quando aplicado no anel dos inteiros de um corpo de números ou em um ideal no anel dos inteiros de um corpo de números é um reticulado no $\mathbb{R}^{n}$. Posteriormente surgiram perturbações deste homomorfismo de modo a obter outras famílias de reticulados. Um outro método ainda pouco explorado de obter reticulados que será apresentado neste trabalho, consiste em obter reticulados via polinômios irredutíveis sobre o corpo dos racionais. Assim, neste trabalho apresentamos construções de versões rotacionadas dos reticulados $A_{2}$ e $D_{3}$, a partir de polinômios $f(x)$ de grau 2 e $g(x)$ de grau 3, respectivamente. Consideramos o caso em que $f(x)$ e $g(x)$ possuem raízes reais e $f(x)$ possui raízes complexas. O método utilizado neste trabalho foi proposto em [1], porém adaptações nas demonstrações de alguns resultados e novos exemplos foram considerados.

\section{Reticulados}

Nesta seção faremos um breve estudo sobre os reticulados, definindo-os e apresentando suas principais propriedades.

Definição 1 Sejam $\left\{v_{1}, v_{2}, \ldots, v_{m}\right\}$ vetores linearmente independentes do $\mathbb{R}^{n}$. O conjunto de pontos

$$
\Lambda=\left\{\mathbf{x}=\sum_{i=1}^{m} \lambda_{i} v_{i}, \lambda_{i} \in \mathbb{Z}\right\}
$$

é chamado reticulado de dimensão $m e\left\{v_{1}, v_{2}, \ldots, v_{m}\right\}$ é chamado de base do reticulado.

Exemplo 2 O reticulado $\Lambda=A^{2}$ é gerado pelos vetores $e_{1}=(1,0), e_{2}=\left(-\frac{1}{2}, \frac{\sqrt{3}}{2}\right)$ e é chamado de reticulado hexagonal.

Definição 3 Seja $\left\{v_{1}, \ldots, v_{m}\right\}$ uma base de um reticulado. O paralelepípedo formado pelos pontos

$$
\lambda_{1} v_{1}+\cdots+\lambda_{m} v_{m}, 0 \leq \lambda_{i}<1
$$

é chamado de paralelepípedo fundamental ou região fundamental do reticulado.

Definição 4 Seja $\left\{v_{1}, \ldots, v_{m}\right\}$ uma base de $\Lambda$. Se $v_{i}=\left(v_{i 1}, \ldots, v_{\text {in }}\right)$, para $i=1, \cdots$, m, a matriz

$$
M=\left(\begin{array}{cccc}
v_{11} & v_{12} & \ldots & v_{1 n} \\
v_{21} & v_{22} & \ldots & v_{2 n} \\
& & \ddots & \\
v_{m 1} & v_{m 2} & \ldots & v_{m n}
\end{array}\right)
$$


é chamada de matriz geradora do reticulado $\Lambda$. A matriz $G=M M^{t}$ é chamada de matriz de Gram do reticulado, onde t denota a transposição.

Assim, os pontos do reticulado são formados por

$$
\Lambda=\left\{\mathbf{x}=\lambda M \mid \lambda \in \mathbb{Z}^{m}\right\} .
$$

Definição 5 O determinante do reticulado $\Lambda$ é definido como sendo o determinante da matriz $G$, ou seja,

$$
\operatorname{det}(\Lambda)=\operatorname{det}(G)
$$

Um reticulado $\Lambda$ é dito ter posto máximo se $m=n$, e neste caso $M$ é uma matriz quadrada. Assim,

$$
\operatorname{det}(\Lambda)=(\operatorname{det}(M))^{2}
$$

Definição 6 Para reticulados de posto máximo, a raiz quadrada do determinante do reticulado é o volume da região fundamental, também chamado volume do reticulado, e denotado por Vol $(\Lambda)$.

É importante observar que o mesmo reticulado pode ser representado por mais de uma matriz, e o fato de dois reticulados terem o mesmo determinante não é suficiente para que eles sejam isomorfos.

Definição 7 Um empacotamento esférico, ou simplesmente um empacotamento no $\mathbb{R}^{n}$, é uma distribuição de esferas de mesmo raio no $\mathbb{R}^{n}$ de forma que a interseção de quaisquer duas esferas tenha no máximo um ponto. Um empacotamento reticulado é um empacotamento em que o conjunto dos centros das esferas formam um reticulado $\Lambda$ no $\mathbb{R}^{n}$. Além disso, $\rho=\min \{|v| ; v \in$ $\Lambda, v \neq 0\} / 2$ é o maior raio para o qual é possível distribuir esferas centradas nos pontos de $\Lambda e$ obter um empacotamento, este raio é então chamado de raio de empacotamento.

Seja $\Lambda \subset \mathbb{R}^{n}$ um reticulado e denotemos por $B(\rho)$ a esfera com centro na origem e raio $\rho$, podemos obter uma expressão para calcular a densidade de empacotamento de $\Lambda$. Temos que

$$
\Delta(\Lambda)=\frac{\text { volume da região coberta por uma esfera }}{\text { volume da região fundamental }}=\frac{\operatorname{Vol}(B(\rho))}{\operatorname{Vol}(\Lambda)}=\frac{\operatorname{Vol}(B(1))(\rho)^{n}}{\operatorname{Vol}(\Lambda)}
$$

Como o valor de $\operatorname{Vol}(B(1))$ é conhecido, podemos reduzir nosso estudo ao cálculo de $\frac{\rho^{n}}{\operatorname{Vol}(\Lambda)}$ que definiremos a seguir.

Definição 8 Seja $\Lambda \subset \mathbb{R}^{n}$ um reticulado. Definimos a densidade de centro de $\Lambda$ por

$$
\delta(\Lambda)=\frac{\rho^{n}}{\operatorname{Vol}(\Lambda)}
$$

onde $\rho$ é o raio de empacotamento de $\Lambda$ e $\operatorname{Vol}(\Lambda)$ seu volume. 
Um dos problemas de empacotamento esférico de um reticulado no $\mathbb{R}^{n}$ é encontrar o empacotamento com maior densidade de centro. Na dimensão um, temos que os pontos de coordenadas inteiras da reta formam um $\mathbb{Z}$-reticulado cuja a densidade de centro é a melhor possível dada por $\delta=1$.

$\mathrm{Na}$ dimensão dois o reticulado hexagonal $A_{2}$ (favo de mel) é o de maior densidade de centro, dada por $\delta=\frac{1}{\sqrt{12}} \approx 0,28868$.

Na dimensão 3 o reticulado conhecido como $f c c$, (face centered cubic) é o de maior densidade de centro, sendo essa $\delta=\frac{1}{\sqrt{32}} \approx 0,17678$.

É conhecido e provado que as densidades de centro dos reticulados $A_{1}, A_{2}, D_{3}$, $D_{4}, D_{5}, E_{6}, E_{7}, E_{8}$ e $\Lambda_{24}$, de dimensões 1 a 8 e 24 , respectivamente, são ótimas. Para outras dimensões não se sabe as ótimas.

\section{Reticulados de dimensão 2 via polinômios de grau 2 com raízes reais}

O objetivo desta seção é apresentar uma construção de reticulados de dimensão 2 via polinômios de grau 2 com duas raízes reais. O Teorema 13 mostrará que é possível obter versões rotacionadas do reticulado $A_{2}$, que como vimos, é o reticulado mais denso na dimensão 2 .

Seja $f(x)=x^{2}+a x+b$ um polinômio de grau 2 mônico e com coeficientes inteiros e sejam $\alpha, \beta \in \mathbb{R}$ as raízes de $f$. Como queremos que $\alpha$ e $\beta$ sejam reais devemos ter

$$
\Delta=a^{2}-4 b>0 \text {. }
$$

A partir das raízes $\alpha$ e $\beta$, podemos definir um reticulado $\Lambda_{f} \subseteq \mathbb{R}^{2}$ gerado pela base $\left\{v_{1}, v_{2}\right\}$, onde $v_{1}=(\alpha, \beta)$ e $v_{2}=(\beta, \alpha)$. Temos que uma matriz geradora de $\Lambda_{f}$ será dada por

$$
M=\left(\begin{array}{cc}
\alpha & \beta \\
\beta & \alpha
\end{array}\right)
$$

Sendo $\rho$ o raio de empacotamento do reticulado $\Lambda_{f}$ segue da Definição 6 e da Definição 8, que a densidade de centro de $\Lambda_{f}$ é dada por

$$
\delta\left(\Lambda_{f}\right)=\frac{\rho^{2}}{\operatorname{Vol}\left(\Lambda_{f}\right)}=\frac{\rho^{2}}{|\operatorname{det}(M)|} .
$$

Assim, para calcularmos a densidade de centro de $\Lambda_{f}$ precisamos encontrar expressões para o cálculo de $\rho$ e de $\operatorname{det}(M)$. O resultado que veremos a seguir nos dá uma expressão para o módulo do determinante da matriz $M$.

Proposição 9 ([1]) Seja $f(x)=x^{2}+a x+b$ um polinômio mônico com coeficientes inteiros $e$ sejam $\alpha$ e $\beta$ as duas raízes reais de $f$. Se $\Lambda_{f}$ é o reticulado com matriz geradora $M$ dada em (1), então o módulo do determinante da matriz $M$ é dado por

$$
|\operatorname{det}(M)|=|a| \sqrt{\Delta}, \text { onde } \Delta=a^{2}-4 b .
$$

Exemplo 10 Sejam $f(x)=x^{2}-4 x+2$ um polinômio de grau 2 com raízes reais $\alpha$ e $\beta$. Temos que $\Delta=(-4)^{2}-4.1 .2=8>0$. Assim, se $\Lambda_{f}$ é o reticulado com base $\{(\alpha, \beta),(\beta, \alpha)\}$ e matriz geradora $M$ como em (1), segue pela Proposição 9 que

$$
|\operatorname{det}(M)|=|a| \sqrt{\Delta}=4 \sqrt{8}=8 \sqrt{2} .
$$


Pela Definição 7, vimos que o maior raio de empacotamento de um reticulado $\Lambda$ é $\rho=$ $\min \{|v| ; v \in \Lambda, v \neq 0\} / 2$. Assim, para que tenhamos um raio de empacotamento máximo devemos encontrar um vetor de $\Lambda_{f}$ cuja norma seja mínima. A seguir, veremos uma expressão para o cálculo da norma de um vetor qualquer de $\Lambda_{f}$.

Proposição 11 Se $f(x)=x^{2}+a x+b \in \mathbb{Z}[x]$ com raízes reais $\alpha$ e $\beta, \Lambda_{f}$ é o reticulado de dimensão 2 gerado pela base $\left\{v_{1}, v_{2}\right\}$, onde $v_{1}=(\alpha, \beta)$ e $v_{2}=(\beta, \alpha)$, e $v \in \Lambda_{f}$, tal que $v=$ $z_{1} v_{1}+z_{2} v_{2}$, com $z_{1}, z_{2} \in \mathbb{Z}$, então,

$$
|v|^{2}=a^{2}\left(z_{1}^{2}+z_{2}^{2}\right)-2 b\left(z_{1}-z_{2}\right)^{2}
$$

Demonstração: Temos que:

$$
|v|^{2}=v \cdot v=z_{1}^{2} v_{1}^{2}+2 z_{1} z_{2} v_{1} v_{2}+z_{2}^{2} v_{2}^{2} .
$$

Observe que, para $i=1,2$, segue que

$$
\begin{aligned}
v_{i}^{2} & =v_{i} \cdot v_{i}=\alpha^{2}+\beta^{2} \\
& =\left(\frac{-a+\sqrt{\Delta}}{2}\right)^{2}+\left(\frac{-a-\sqrt{\Delta}}{2}\right)^{2} \\
& =\frac{a^{2}-2 a \sqrt{\Delta}+\Delta}{4}+\frac{a^{2}+2 a \sqrt{\Delta}+\Delta}{4} \\
& =\frac{a^{2} \Delta}{2}=a^{2}-2 b,
\end{aligned}
$$

$\mathrm{e}$

$$
\begin{aligned}
v_{1} \cdot v_{2} & =2 \alpha \beta \\
& =2\left(\frac{-a+\sqrt{\Delta}}{2}\right) \cdot\left(\frac{-a-\sqrt{\Delta}}{2}\right) \\
& =\frac{a^{2}-\Delta}{2}=2 b
\end{aligned}
$$

Assim,

$$
\begin{aligned}
|v|^{2} & =\left(a^{2}-2 b\right)\left(z_{1}^{2}+z_{2}^{2}\right)+2(2 b) z_{1} z_{2} \\
& =a^{2}\left(z_{1}^{2}+z_{2}^{2}\right)-2 b\left(z_{1}^{2}+z_{2}^{2}-z_{1} z_{2}\right) \\
& =a^{2}\left(z_{1}^{2}+z_{2}^{2}\right)-2 b\left(z_{1}-z_{2}\right)^{2}
\end{aligned}
$$

como queríamos .

Agora que temos uma expressão para calcular a norma de um vetor de $\Lambda_{f}$ resta saber onde este vetor atinge norma mínima e daí teremos o raio de empacotamento do reticulado e portanto podemos calcular efetivamente a densidade de centro do reticulado $\Lambda_{f}$. Nestas condições, a densidade de centro de $\Lambda_{f}$ é dada por

$$
\delta\left(\Lambda_{f}\right)=\frac{\left(\frac{\sqrt{\psi_{1}}}{2}\right)^{2}}{|a| \sqrt{\Delta}},
$$

onde $\psi_{1}=\min \left\{v=a^{2}\left(z_{1}^{2}+z_{2}^{2}\right)-2 b\left(z_{1}-z_{2}\right)^{2} \mid z_{1}, z_{2} \in \mathbb{Z}\right\}$. 
Exemplo 12 Nas condições do Exemplo 10, seja $v=z_{1} v_{1}+z_{2} v_{2} \in \Lambda_{f}$. Pela Proposição 11 segue que

$$
|v|^{2}=16\left(z_{1}^{2}+z_{2}^{2}\right)-4\left(z_{1}-z_{2}\right)^{2}
$$

e, este vetor assume valor mínimo 12 quando tomamos $z_{1}=1$ e $z_{2}=0$. Logo, a densidade de centro do reticulado $\Lambda_{f}$ é dada por

$$
\delta\left(\Lambda_{f}\right)=\frac{\left(\frac{\sqrt{12}}{2}\right)^{2}}{8 \sqrt{2}}=\frac{3}{8 \sqrt{2}} \cong 0,2651 .
$$

Vimos que o reticulado $A_{2}$ é o reticulado mais denso na dimensão 2, cuja densidade de centro é $\delta\left(A_{2}\right) \cong 0,28868$. No resultado a seguir apresentamos uma condição sobre os coeficientes do polinômio $f$ para que o reticulado $\Lambda_{f}$ tenha a mesma densidade de centro do reticulado $A_{2}$.

Teorema 13 ([1]) Sejam $f(x)=x^{2}+a x+b \in \mathbb{Z}[x]$ com raízes reais $\alpha$ e $\beta, \Lambda_{f}$ o reticulado de dimensão 2 gerado pela base $\left\{v_{1}, v_{2}\right\}$, onde $v_{1}=(\alpha, \beta)$ e $v_{2}=(\beta, \alpha)$. Se $\frac{a^{2}}{6}=b$, então $\Lambda_{f}$ possui a maior densidade de centro possível para dimensão 2.

Demonstração: Seja $f(x)=x^{2}+a x+b, a, b \in \mathbb{Z}$ tal que $\frac{a^{2}}{6}=b$ e seja $v=z_{1} v_{1}+z_{2} v_{2}$, com $z_{1}, z_{2} \in \mathbb{Q}$ um vetor de $\Lambda_{f}$. Pela Proposição 11 temos que

$$
\begin{aligned}
|v|^{2} & =a^{2}\left(z_{1}^{2}+z_{2}^{2}\right)-2 b\left(z_{1}-z_{2}\right)^{2} \\
& =6 b\left(z_{1}{ }^{2}+z_{2}^{2}\right)-2 b\left(z_{1}-z_{2}\right)^{2} .
\end{aligned}
$$

Observe que esta forma quadrática assume valor mínimo $4 b$ quando $z_{1}=1$ e $z_{2}=0$. Logo,

$$
\psi_{1}=\min \left\{v=6 b\left(z_{1}^{2}+z_{2}^{2}\right)-2 b\left(z_{1}-z_{2}\right)^{2} \mid z_{1}, z_{2} \in \mathbb{Z}\right\}=4 b .
$$

Agora, pela Proposição 9 temos

$$
|\operatorname{det}(M)|=|a| \sqrt{a^{2}-4 b}=\sqrt{6 b} \sqrt{2 b}=2 \sqrt{3} b .
$$

Logo, por (2), (3) e (4) segue que a densidade de centro de $\Lambda_{f}$ será dada por

$$
\delta\left(\Lambda_{f}\right)=\frac{\left(\frac{\sqrt{4 b}}{2}\right)^{2}}{2 \sqrt{3} b}=\frac{b}{2 \sqrt{3} b}=\frac{1}{2 \sqrt{3}} \cong 0,28868
$$

que é a mesma densidade de centro do reticulado $A_{2}$. Portanto, $\Lambda_{f}$ possui densidade de centro ótima para dimensão 2 .

Observe que a partir do Teorema 13, obtemos uma família de reticulados de dimensão 2 com densidade de centro ótima, basta que o coeficiente $a$ do polinômio $f(x)=x^{2}+a x+b$ divida 6 , ou seja, tomando $a= \pm 6,12,18,24, \ldots$ teremos a condição satisfeita. A seguir, explicitamos esta construção para $a=-6$ e $a=12$.

Exemplo 14 Sejam $f(x)=x^{2}-6 x+6, \alpha, \beta \in \mathbb{R}$ as raízes de $f$ e $v=z_{1} v_{1}+z_{2} v_{2} \in \Lambda_{f}$, onde $v_{1}=(\alpha, \beta)$ e $v_{2}=(\beta, \alpha)$. Temos que

$$
|v|^{2}=36\left(z_{1}^{2}+z_{2}^{2}\right)-12\left(z_{1}-z_{2}\right)^{2}
$$


que assume o valor mínimo 24. Temos ainda que

$$
|\operatorname{det}(M)|=|a| \sqrt{a^{2}-4 b}=6 \sqrt{12}=12 \sqrt{3} .
$$

Portanto,

$$
\delta\left(\Lambda_{f}\right)=\frac{\left(\frac{\sqrt{24}}{2}\right)}{12 \sqrt{3}}=\frac{24}{48 \sqrt{3}}=\frac{1}{2 \sqrt{3}},
$$

que é a densidade ótima para dimensão 2.

Exemplo $15 \operatorname{Sejam} f(x)=x^{2}+12 x+24, \alpha, \beta \in \mathbb{R}$ as raizes de $f$ e $v=z_{1} v_{1}+z_{2} v_{2} \in \Lambda_{f}$, $v_{1}=$ $(\alpha, \beta)$ e $v_{2}=(\beta, \alpha)$. Temos que

$$
|v|^{2}=144\left(z_{1}^{2}+z_{2}^{2}\right)-48\left(z_{1}-z_{2}\right)^{2}
$$

assume valor mínimo 48. Temos ainda que,

$$
|\operatorname{det}(M)|=|a| \sqrt{a^{2}-4 b}=12 \sqrt{12}=24 \sqrt{3} .
$$

Com isso

$$
\delta\left(\Lambda_{f}\right)=\frac{\left(\frac{\sqrt{48}}{2}\right)}{12 \sqrt{3}}=\frac{12}{2 \sqrt{3}}=\frac{1}{2 \sqrt{3}},
$$

que é a densidade ótima para dimensão 2.

\section{Reticulados de dimensão 2 via polinômios de grau 2 com raízes complexas conjugadas}

O objetivo desta seção é apresentar uma construção de reticulados de dimensão 2 via polinômios de grau 2 com duas raízes complexas conjugadas. O Teorema 20 mostrará que é possível obter reticulados que são versões rotacionadas do reticulado $A_{2}$ utilizando tais polinômios.

Seja $f(x)=x^{2}+a x+b$, um polinômio mônico com coeficientes inteiros e $\gamma_{1}, \gamma_{2} \in \mathbb{C}$ as raízes de $f$. Como queremos que $f$ não tenha reais devemos ter

$$
\Delta=a^{2}-4 b<0 .
$$

Consideremos $\gamma_{1}=\alpha+i \beta$ e $\gamma_{2}=\alpha-i \beta$, com $\alpha, \beta \in \mathbb{R}$ e $\beta \neq 0$ as raízes de $f$. Podemos definir $\Lambda_{f} \subset \mathbb{R}^{2}$ um reticulado gerado pela base $\left\{v_{1}, v_{2}\right\}$, onde $v_{1}=(\alpha, \beta)$ e $v_{2}=(\alpha,-\beta)$. Temos que uma matriz geradora de $\Lambda_{f}$ será dada por

$$
M=\left(\begin{array}{cc}
\alpha & \beta \\
\alpha & -\beta
\end{array}\right)
$$

Da mesma forma como no caso anterior, queremos encontrar expressões para o calcular $\rho$ e $\operatorname{det}(M)$, para poder calcular a densidade de centro destes reticulados. No resultado a seguir veremos uma expressão para o cálculo de $|\operatorname{det}(M)|$.

Proposição 16 ([1]) Se $f(x)=x^{2}+a x+b \in \mathbb{Z}[x]$ com raízes complexas $\alpha \pm i \beta, \alpha, \beta \in \mathbb{R}, \beta \neq 0$ e M é a matriz dada em (5), então o módulo do determinante de $M$ é dado por

$$
|\operatorname{det}(M)|=\frac{|a| \sqrt{-\Delta}}{2}, \text { onde } \Delta=a^{2}-4 b<0 .
$$


Exemplo 17 Sejam $f(x)=x^{2}+x+2$ um polinômio de grau 2 com raízes complexas conjugadas $\alpha+i \beta, \alpha-i \beta \in \mathbb{C}$. Temos que: $\Delta=1^{2}-4.1 .2=-7<0$. Assim, se $\Lambda_{f}$ é o reticulado com base $\{(\alpha, \beta),(\alpha,-\beta)\}$ e matriz geradora $M$, segue pela Proposição 16 que

$$
|\operatorname{det}(M)|=\frac{|a| \sqrt{-\Delta}}{2}=\frac{\sqrt{7}}{2} .
$$

Vejamos agora um resultado que nos dá uma expressão para o cálculo da norma de um vetor $\operatorname{de} \Lambda_{f}$.

Proposição 18 ([1]) Se $f(x)=x^{2}+a x+b \in \mathbb{Z}[x]$ com raízes complexas conjugadas $\alpha \pm i \beta, \alpha, \beta \in$ $\mathbb{R}, \beta \neq 0, \Lambda_{f}$ é o reticulado de dimensão 2 gerado pela base $\left\{v_{1}, v_{2}\right\}$, onde $v_{1}=(\alpha, \beta) e$ $v_{2}=(\alpha,-\beta)$, e $v \in \Lambda_{f}$, tal que $v=z_{1} v_{1}+z_{2} v_{2}$, com $z_{1}, z_{2} \in \mathbb{Q}$, então,

$$
|v|^{2}=\frac{a^{2}}{4}\left(z_{1}+z_{2}\right)^{2}+\frac{4 b-a^{2}}{4}\left(z_{1}-z_{2}\right)^{2} .
$$

Demonstração: Sejam $\gamma_{1}=\alpha+i \beta$ e $\gamma_{2}=\alpha-i \beta$, com $\alpha, \beta \in \mathbb{R}$ e $\beta \neq 0$ as raízes de $f$ e $v=z_{1} v_{1}+z_{2} v_{2}$, com $v_{1}=(\alpha, \beta), v_{2}=(\alpha,-\beta)$ e $z_{1}, z_{2} \in \mathbb{Q}$. Temos que

$$
v=z_{1}(\alpha, \beta)+z_{2}(\alpha,-\beta)=\left(\alpha\left(z_{1}+z_{2}\right), \beta\left(z_{1}-z_{2}\right)\right) .
$$

Logo,

$$
\begin{aligned}
|v|^{2} & =\alpha^{2}\left(z_{1}+z_{2}\right)^{2}+\beta^{2}\left(z_{1}-z_{2}\right)^{2} \\
& =\left(\frac{-a}{2}\right)^{2}\left(z_{1}+z_{2}\right)^{2}+\left(\frac{\sqrt{-\Delta}}{2}\right)^{2}\left(z_{1}-z_{2}\right)^{2} \\
& =\frac{a^{2}}{4}\left(z_{1}+z_{2}\right)^{2}+\frac{4 b-a^{2}}{4}\left(z_{1}-z_{2}\right)^{2},
\end{aligned}
$$

como queríamos mostrar.

A partir das Proposições 16 e 18, a densidade de centro do reticulado $\Lambda_{f}$ é dada por

$$
\delta\left(\Lambda_{f}\right)=\frac{\left(\frac{\sqrt{\psi_{2}}}{2}\right)^{2}}{\frac{a \sqrt{-\Delta}}{2}}
$$

onde $\psi_{2}=\min \left\{v=\frac{a^{2}}{4}\left(z_{1}+z_{2}\right)^{2}+\frac{4 b-a^{2}}{4}\left(z_{1}-z_{2}\right)^{2} \mid z_{1}, z_{2} \in \mathbb{Z}\right\}$.

Exemplo 19 Nas condições do Exemplo 17, seja $v=z_{1} v_{1}+z_{2} v_{2} \in \Lambda_{f}$. Pela Proposição 18 segue que

$$
|v|^{2}=\frac{\left(z_{1}+z_{2}\right)^{2}+7\left(z_{1}-z_{2}\right)^{2}}{4}
$$

e, este vetor assume o valor mínimo 1 quando tomamos $z_{1}=1$ e $z_{2}=1$. Portanto, a densidade de centro do reticulado $\Lambda_{f}$ é dada por

$$
\delta\left(\Lambda_{f}\right)=\frac{\left(\frac{1}{2}\right)^{2}}{\left|\frac{\sqrt{7}}{2}\right|}=\frac{1}{2 \sqrt{7}} \cong 0,18898
$$


Com o seguinte teorema, temos novamente um família de reticulados de posto 2 com densidade de centro ótima.

Teorema 20 ([1]) Sejam $f(x)=x^{2}+a x+b \in \mathbb{Z}[x]$ com raízes complexas conjugadas $\alpha \pm i \beta, \alpha, \beta \in$ $\mathbb{R}, \beta \neq 0, \Lambda_{f}$ o reticulado de dimensão 2 gerado pela base $\left\{v_{1}, v_{2}\right\}$, onde $v_{1}=(\alpha, \beta)$ e $v_{2}=$ $(\alpha,-\beta)$. Se $f$ satisfaz a condição $a^{2}=b$, então o reticulado $\Lambda_{f}$ possui densidade de centro ótima.

Demonstração: Seja $f(x)=x^{2}+a x+b, a, b \in \mathbb{Z}$ tal que $a^{2}=b$ e seja $v=z_{1} v_{1}+z_{2} v_{2}$, com $z_{1}, z_{2} \in \mathbb{Q}$ um vetor de $\Lambda_{f}$. Pela Proposição 18 temos que

$$
\begin{aligned}
|v|^{2} & =\frac{a^{2}}{4}\left(z_{1}+z_{2}\right)^{2}+\frac{4 b-a^{2}}{4}\left(z_{1}-z_{2}\right)^{2} \\
& =\frac{b}{4}\left(z_{1}+z_{2}\right)^{2}+\frac{3 b}{4}\left(z_{1}-z_{2}\right)^{2} .
\end{aligned}
$$

Observe que esta forma quadrática assume valor mínimo $b$ quando $z_{1}=1$ e $z_{2}=0$. Logo,

$$
\psi_{2}=\min \left\{v=\frac{a^{2}}{4}\left(z_{1}+z_{2}\right)^{2}+\frac{4 b-a^{2}}{4}\left(z_{1}-z_{2}\right)^{2} \mid z_{1}, z_{2} \in \mathbb{Z}\right\}=b .
$$

Agora, pela Proposição 16 temos

$$
|\operatorname{det}(M)|=\frac{|a| \sqrt{-\left(a^{2}-4 b\right)}}{2}=\sqrt{b} \sqrt{3 b}=b \sqrt{3} .
$$

Por, (6), (7) e (8) segue que a densidade de centro de $\Lambda_{f}$ será dada por

$$
\delta\left(\Lambda_{f}\right)=\frac{\left(\frac{\sqrt{b}}{2}\right)^{2}}{b \sqrt{3}}=\frac{b}{2 b \sqrt{3}}=\frac{1}{2 \sqrt{3}} \cong 0,28868,
$$

que é a mesma densidade de centro do reticulado $A_{2}$. Portanto, $\Lambda_{f}$ possui densidade de centro ótima para dimensão 2.

Como vimos no Teorema 20, fazendo $a^{2}=b$ no polinômio $f(x)=x^{2}+a x+b$ com raízes complexas conjugadas obtemos uma família de reticulados em dimensão 2 com a mesma densidade de centro do reticulado $A_{2}$. A seguir explicitaremos esta construção para $a=-2$.

Exemplo 21 Sejam $f(x)=x^{2}-2 x+4, \alpha \pm i \beta \in \mathbb{C}$ as raízes de $f$ e $v=z_{1} v_{1}+z_{2} v_{2} \in \Lambda_{f}$, onde $v_{1}=(\alpha, \beta)$ e $v_{2}=(\alpha,-\beta)$. Temos que

$$
|v|^{2}=\frac{2^{2}}{4}\left(z_{1}+z_{2}\right)^{2}+\frac{4.4-4}{4}\left(z_{1}-z_{2}\right)^{2},
$$

que assume o valor mínimo 4, quando $z_{1}=1$ e $z_{2}=0$. Além disso,

$$
|\operatorname{det}(M)|=\frac{|a| \sqrt{-\left(a^{2}-4 b\right)}}{2}=\frac{|-2| \sqrt{12}}{2}=\sqrt{12} .
$$

Portanto,

$$
\delta\left(\Lambda_{f}\right)=\frac{\left(\frac{\sqrt{4}}{2}\right)}{\sqrt{12}}=\frac{1}{\sqrt{12}} \cong 0,28868
$$

que é a densidade ótima para dimensão 2 . 


\section{Reticulados de dimensão 3 via polinômios de grau 3 com raízes reais}

O objetivo desta seção é apresentar uma construção de reticulados de dimensão 3 via polinômios de grau 3 com 3 raízes reais. O Teorema 27 mostrará que é possível obter reticulados que são versões rotacionadas do reticulado $D_{3}$ via tais polinômios, que como vimos é o reticulado com a melhor densidade de centro na dimensão 3.

Seja $f(x)=x^{3}+a x^{2}+b x+c$ um polinômio mônico com coeficientes inteiros e sejam $\alpha, \beta, \gamma \in$ $\mathbb{C}$ as raízes de $f$. Queremos que $f$ possua somente raízes reais. Iniciamos mostrando um resultado que nos garante esta condição.

Proposição 22 ([1]) Seja $f(x)=x^{3}+a x^{2}+b x+c$ um polinômio mônico com coeficientes inteiros. Para que as raízes de $f$ sejam reais é necessário e suficiente que

$$
a^{2}-3 b>0 \text { e }\left(\sqrt{a^{2}-3 b}\right)^{3}>\left|\frac{2 a^{2}-9 a b+27 c}{2}\right| .
$$

Demonstração: Uma condição necessária e suficiente para que as raízes de $f$ sejam todas reais é que sua derivada se anule em dois pontos distintos e que a função $f$ aplicada nestes pontos tenham sinais distintos. Assim, se $f(x)=x^{3}+a x^{2}+b x+c, \operatorname{com} a, b, c \in \mathbb{Z}$ temos que sua derivada é dada por $f^{\prime}(x)=3 x^{2}+2 a x+b$ cujas raízes são

$$
x_{1}=\frac{-a-\sqrt{a^{2}-3 b}}{3} \text { e } x_{2}=\frac{-a+\sqrt{a^{2}-3 b}}{3} .
$$

Daí, segue que $a^{2}-3 b>0$ deve ser um número positivo. Agora,

$$
\begin{aligned}
f\left(x_{1}\right) & =x_{1}^{2}+a x_{1}^{2}+b x_{1}+c \\
& =\left(\frac{-a-\sqrt{a^{2}-3 b}}{3}\right)^{3}+a\left(\frac{-a-\sqrt{a^{2}-3 b}}{3}\right)^{2}+b\left(\frac{-a-\sqrt{a^{2}-3 b}}{3}\right)+c \\
& =\frac{1}{27}\left(2 a^{3}+2\left(\sqrt{a^{2}-3 b}\right)^{3}-9 a b+27 c\right)
\end{aligned}
$$

e, portanto, $f\left(x_{1}\right)>0$ se, e somente se,

$$
\left(\sqrt{a^{2}-3 b}\right)^{3}>\frac{2 a^{2}-9 a b+27 c}{2} .
$$

Analogamente,

$$
f\left(x_{2}\right)=\frac{1}{27}\left(2 a^{3}+2\left(\sqrt{a^{2}-3 b}\right)^{3}-9 a b+27 c\right)
$$

e, portanto, $f\left(x_{2}\right)<0$ se, e somente se,

$$
\left(\sqrt{a^{2}-3 b}\right)^{3}>\frac{2 a^{2}-9 a b+27 c}{2} .
$$

Logo, para que as raízes de $f$ sejam reais devemos ter

$$
a^{2}-3 b>0 \text { e }\left(\sqrt{a^{2}-3 b}\right)^{3}>\left|\frac{2 a^{2}-9 a b+27 c}{2}\right|
$$


como queríamos.

Sejam $\alpha, \beta, \gamma \in \mathbb{R}$ as raízes de $f(x)=x^{3}+a x^{2}+b x+c \in \mathbb{Z}[x]$ que satisfazem as condições da Proposição 22. Podemos definir $\Lambda_{f} \subset \mathbb{R}^{3}$ como o reticulado gerado pela base $\left\{v_{1}, v_{2}, v_{3}\right\}$, onde $v_{1}=(\alpha, \beta, \gamma), v_{2}=(\gamma, \alpha, \beta)$ e $v_{3}=(\beta, \gamma, \alpha)$. Temos que uma matriz geradora de $\Lambda_{f}$ será

$$
M=\left(\begin{array}{lll}
\alpha & \beta & \gamma \\
\gamma & \alpha & \beta \\
\beta & \gamma & \alpha
\end{array}\right)
$$

e sua densidade de centro será dada por:

$$
\delta\left(\Lambda_{f}\right)=\frac{\rho^{3}}{|\operatorname{det}(M)|},
$$

onde $\rho$ é o raio de empacotamento de $\Lambda_{f}$.

Da mesma forma como para dimensão 2, queremos encontrar expressões para o calcular $\rho$ e $\operatorname{det}(M)$. No resultado a seguir veremos uma expressão para o cálculo de $\operatorname{det}(M)$.

Proposição 23 ([1]) Seja $f(x)=x^{3}+a x^{2}+b x+c$ um polinômio mônico com coeficientes inteiros e sejam $\alpha, \beta, \gamma$ as raízes de $f$, satisfazendo a condição da Proposição 22. Se $\Lambda_{f}$ é o reticulado obtido a partir de f com matriz geradora $M$ como dada em (9), então o módulo do determinante de $M$ é dado por

$$
|\operatorname{det}(M)|=\left|a\left(a^{2}-3 b\right)\right| \text {. }
$$

Demonstração: Temos que o determinante de $M$ será dado por

$$
\operatorname{det}(M)=\alpha^{3}+\beta^{3}+\gamma^{3}-3 \alpha \beta \gamma
$$

Das relações de Girard segue que:

$$
\left\{\begin{array}{c}
\alpha+\beta+\gamma=-a \\
\alpha \beta+\alpha \gamma+\beta \gamma=b \\
\beta \gamma \alpha=-c
\end{array}\right.
$$

assim,

$$
(\alpha+\beta+\gamma)^{2}=\alpha^{2}+\beta^{2}+\gamma^{2}+2(\alpha \beta+\alpha \gamma+\beta \gamma)=a^{2}
$$

$\log 0$

$$
\alpha^{2}+\beta^{2}+\gamma^{2}=a^{2}-2 b
$$

Como $\alpha+\beta+\gamma=-a$, multiplicando o lado esquerdo da Equação (10) por $\alpha+\beta+\gamma$ e o lado direito por $-a$ teremos

$$
\begin{aligned}
& \alpha^{3}+\beta^{3}+\gamma^{3}+\alpha \beta^{2}+\beta \alpha^{2}+\beta \gamma^{2}+\gamma \alpha^{2}+\gamma \beta^{2} \\
= & \alpha^{3}+\beta^{3}+\gamma^{3}+\alpha \beta(\alpha+\beta)+\alpha \gamma(\alpha+\gamma)+\beta \gamma(\beta+\gamma) \\
= & \alpha^{3}+\beta^{3}+\gamma^{3}-\alpha \beta(\gamma+a)-\alpha \gamma(\beta+a)-\beta \gamma(\alpha+a) \\
= & \alpha^{3}+\beta^{3}+\gamma^{3}-3 \alpha \beta \gamma-a(\alpha \beta+\alpha \gamma+\beta \gamma) \\
= & -a\left(a^{2}-2 b\right) .
\end{aligned}
$$

Logo,

$$
\alpha^{3}+\beta^{3}+\gamma^{3}-3 \alpha \beta \gamma=-a\left(a^{2}-2 b\right)+a b=-a^{3}+3 a b .
$$

Portanto, $|\operatorname{det}(M)|=\left|a\left(a^{2}-3 b\right)\right|$ o que prova a proposição. 
Exemplo 24 Sejam $f(x)=x^{3}-9 x^{2}+23 x-15$ um polinômio de grau 3 com raízes reais $\alpha, \beta, \gamma$. Se $\Lambda_{f}$ é o reticulado com base $\left\{v_{1}, v_{2}, v_{3}\right\}$ e matriz geradora $M$, segue pela Proposição 23 que

$$
|\operatorname{det}(M)|=\left|a\left(a^{2}-3 b\right)\right|=108 .
$$

Agora, veremos um resultado que nos dará uma expressão para o cálculo da norma de um vetor de $\Lambda_{f}$.

Proposição 25 ([1]) Seja $f(x)=x^{3}+a x^{2}+b x+c$ um polinômio mônico com coeficientes inteiros e sejam $\alpha, \beta, \gamma$ as raízes de $f$, satisfazendo a condição da Proposição 22. Se $\Lambda_{f}$ é o reticulado de dimensão 3 gerado pela base $\left\{v_{1}, v_{2}, v_{3}\right\}$, onde $v_{1}=(\alpha, \beta, \gamma), v_{2}=(\gamma, \alpha, \beta), v_{3}=(\beta, \gamma, \alpha), e$ $v \in \Lambda_{f}$, tal que $v=z_{1} v_{1}+z_{2} v_{2}+z_{3} v_{3}$, com $z_{1}, z_{2}, z_{3} \in \mathbb{Q}$. Então,

$$
|v|^{2}=\left(a^{2}-2 b\right)\left(z_{1}^{2}+z_{2}^{2}+z_{3}^{2}\right)+2 b\left(z_{1} z_{2}+z_{1} z_{3}+z_{2} z_{3}\right) .
$$

A partir das Proposições 23 e 25, a densidade de centro do reticulado $\Lambda_{f}$ é dada por

$$
\delta\left(\Lambda_{f}\right)=\frac{\left(\frac{\sqrt{\Psi_{3}}}{2}\right)^{3}}{\left|a\left(a^{2}-3 b\right)\right|},
$$

onde $\psi_{3}=\min \left\{v=\left(a^{2}-2 b\right)\left(z_{1}^{2}+z_{2}^{2}+z_{3}^{2}\right)+2 b\left(z_{1} z_{2}+z_{2} z_{3}+z_{1} z_{3}\right) \mid z_{1}, z_{2}, z_{3} \in \mathbb{Z}\right\}$.

Exemplo 26 Nas condições do Exemplo 24, seja $v=z_{1} v_{1}+z_{2} v_{2}+z_{3} v_{3} \in \Lambda_{f}$. Pela Proposição 25 segue que

$$
|v|^{2}=35\left(z_{1}^{2}+z_{2}^{2}+z_{3}^{2}\right)+46\left(z_{1} z_{2}+z_{1} z_{3}+z_{2} z_{3}\right)
$$

e, este vetor assume o valor mínimo 24 quando tomamos $z_{1}=1, z_{2}=-1$ e $z_{3}=0$. Portanto, a densidade de centro do reticulado $\Lambda_{f}$ é dada por

$$
\delta\left(\Lambda_{f}\right)=\frac{(\sqrt{6})^{3}}{108}=\frac{\sqrt{6}}{18}
$$

Teorema 27 ([1]) Seja $f(x)=x^{3}+a x^{2}+b x+c$ um polinômio mônico com coeficientes inteiros e sejam $\alpha, \beta, \gamma$ as raízes de $f$, satisfazendo a condição da Proposição 22. Se $f$ satisfaz

$$
\left(\frac{a}{2}\right)^{2}=b \text { e } c\left(27 c+4 a^{3}-18 a b\right)<0,
$$

então o reticulado $\Lambda_{f}$ gerado pela base $\left\{v_{1}, v_{2}, v_{3}\right\}$, onde $v_{1}=(\alpha, \beta, \gamma), v_{2}=(\gamma, \alpha, \beta)$ e $v_{3}=$ $(\beta, \gamma, \alpha)$, possui densidade de centro ótima.

Demonstração: Pela Proposição (25) temos que

$$
\begin{aligned}
|v|^{2} & =\left(a^{2}-2 b\right)\left(z_{1}^{2}+z_{2}^{2}+z_{3}^{2}\right)+2 b\left(z_{1} z_{2}+z_{1} z_{3}+z_{2} z_{3}\right) \\
& =2 b\left(z_{1}^{2}+z_{2}^{2}+z_{3}^{2}+z_{1} z_{2}+z_{1} z_{3}+z_{2} z_{3}\right) .
\end{aligned}
$$

Observe que esta forma quadrática assume valor mínimo $2 b$ quando $z_{1}=1$ e $z_{2}=z_{3}=0$. Logo,

$$
\psi_{3}=\min \left\{v=\left(a^{2}-2 b\right)\left(z_{1}^{2}+z_{2}^{2}+z_{3}^{2}\right)+2 b\left(z_{1} z_{2}+z_{2} z_{3}+z_{1} z_{3}\right) \mid z_{1}, z_{2}, z_{3} \in \mathbb{Z}\right\}=2 b .
$$


Agora, pela Proposição 23, temos

$$
|\operatorname{det}(M)|=\left|a\left(a^{2}-3 b\right)\right|=|a b| .
$$

Por, (11), (12) e (13) segue que a densidade de centro de $\Lambda_{f}$ será dada por

$$
\delta\left(\Lambda_{f}\right)=\frac{\left(\frac{\sqrt{2 b}}{2}\right)^{3}}{|a b|}=\frac{1}{4 \sqrt{2}} \cong 0,17678,
$$

que é a mesma densidade de centro do reticulado $D_{3}$. Portanto, $\Lambda_{f}$ possui densidade de centro ótima para dimensão 3 .

Exemplo 28 Sejam $f(x)=x^{3}-6 x^{2}+9 x-1$ com raízes reais $\alpha, \beta, \gamma, \Lambda_{f}$ um reticulado de dimensão 3 gerado pela base $\left\{v_{1}, v_{2}, v_{3}\right\}$, onde $v_{1}=(\alpha, \beta, \gamma), v_{2}=(\gamma, \alpha, \beta), v_{3}=(\beta, \gamma, \alpha)$, e $v \in \Lambda_{f}$, tal que $v=z_{1} v_{1}+z_{2} v_{2}+z_{3} v_{3}$, com $z_{1}, z_{2}, z_{3} \in \mathbb{Q}$. Temos que

$$
|v|^{2}=18\left(z_{1}^{2}+z_{2}^{2}+z_{3}^{2}\right)+18\left(z_{1} z_{2}+z_{1} z_{3}+z_{2} z_{3}\right),
$$

que assume o valor mínimo 18 , quando $z_{1}=1$ e $z_{2}=z_{3}=0$. Temos ainda que

$$
|\operatorname{det}(M)|=\left|a\left(a^{2}-3 b\right)\right|=54 .
$$

Portanto,

$$
\delta\left(\Lambda_{f}\right)=\frac{\frac{3 \sqrt{2}}{2}}{54}=\frac{\sqrt{2}}{8}=\frac{1}{4 \sqrt{2}} \cong 0,17678,
$$

que é a densidade de centro ótima para essa dimensão.

\section{Considerações Finais}

Neste trabalho foi apresentado uma maneira de se construir os reticulados $A_{2}$ e $D_{3}$ via polinômios. Existem outras maneiras de se construir tais reticulados, por exemplo, através do homomorfismo de Minkowski, entretanto, construir reticulados utilizando polinônios ainda é um método pouco explorado na literatura e devido as propriedades existentes sobre as raízes de polinômios, sua construção se torna mais simples do que a construção via o homomorfismo de Minkowski que utiliza corpo de números. Na dimensão 3 por exemplo, é preciso considerar subcorpos de corpos ciclotômicos para utilizar o homomorfismo de Minkowski. Acreditamos que, da mesma forma como foram obtidos os reticulados mais densos nas dimensões 2 e 3 via polinômios, pode-se obter reticulados com densidade de centro ótima para dimensões maiores.

\section{Referências Bibliográficas}

[1] SOUZA, T. M. Reticulados algébricos em corpos de números abelianos. 2004, 119 f. Dissertação (Mestrado em Matemática) - Universidade Estadual Paulista "Júlio de Mesquita Filho”, São José do Rio Preto, 2004.

[2] BENEDITO, C. W. O. Famílias de reticulados algébricos e reticulados ideais. 2010. 165 f. Dissertação (Mestrado em Matemática) - Universidade Estadual Paulista "Júlio de Mesquita 
Filho”, São José do Rio Preto, 2010.

[3] BOUTROS, J. et al. Good lattice constellations for both Rayleigh fading and Gaussian channels. IEEE Transactions on Information Theory, v. 42, n. 2, p. 502-518, 1996.

[4] CONWAY, J. H.; SLOANE, N. J. A. Sphere packings, lattices and groups. New York: Springer-Verlag, 1988.

[5] PINTO, W. L. S.; ALVES, C.; BENEDITO, C. W. O. Reticulados via polinômios de grau 2 e 3. In: ENCONTRO REGIONAL DE MATEMÁTICA APLICADA E COMPUTACIONAL, 4., 2017, Bauru. Caderno de trabalhos completos e resumos. Bauru: Unesp, Faculdade de Ciências, 2017. p. 532-535. Disponível em:

$<$ http://www.fc.unesp.br/\#!/departamentos/matematica/eventos2341/ermac/cadesnos-de-trabalhoscompletos-e-resumos/>. Acesso em: 10 out. 2017.

[6] SAMUEL, P. Algebraic theory of numbers. Paris: Hermann, 1970.

[7] STEWART, I. N.; TALL, D. O. Algebraic number theory. 2. ed. London: Chapman \& Hall, 1987.

Artigo recebido em jun. 2017 e aceito em nov. 2017. 